\title{
Kinetic approach to a relativistic BEC with inelastic processes
}

\author{
Richard Lenkiewicz, ${ }^{1, *}$ Alex Meistrenko๑, ${ }^{1}$ Hendrik van Hees, ${ }^{1, \dagger}$ Kai Zhou $\odot,{ }^{1,2}$ Zhe Xu®,${ }^{3}$ and Carsten Greiner ${ }^{1}$ \\ ${ }^{1}$ Institut für Theoretische Physik, Goethe-Universität Frankfurt, \\ Max-von-Laue-Straße 1, D-60438 Frankfurt am Main, Germany \\ ${ }^{2}$ Frankfurt Institute for Advanced Studies, Ruth-Moufang-Straße 1, D-60438 Frankfurt am Main, Germany \\ ${ }^{3}$ Department of Physics, Tsinghua University and Collaborative Innovation Center of Quantum Matter, \\ Beijing 100084, China
}

(Received 9 July 2019; published 11 November 2019)

\begin{abstract}
The phenomenon of Bose-Einstein condensation is investigated in the context of the color-glasscondensate description of the initial state of ultrarelativistic heavy-ion collisions. For the first time, in this paper, we study the influence of particle-number changing $2 \leftrightarrow 3$ processes on the transient formation of a Bose-Einstein condensate within an isotropic system of scalar bosons by including $2 \leftrightarrow 3$ interactions of massive bosons with constant and isotropic cross sections, following a Boltzmann equation. The oneparticle distribution function is decomposed in a condensate part and a nonzero momentum part of excited modes, leading to coupled integro-differential equations for the time evolution of the condensate and phasespace distribution function, which are then solved numerically. Our simulations converge to the expected equilibrium state, and only for $\sigma_{23} / \sigma_{22} \ll 1$, we find that a Bose-Einstein condensate emerges and decays within a finite lifetime in contrast to the case where only binary scattering processes are taken into account, and the condensate is stable due to particle-number conservation. Our calculations demonstrate that BoseEinstein condensates in the very early stage of heavy-ion collisions are highly unlikely, if inelastic collisions are significantly participating in the dynamical gluonic evolution.
\end{abstract}

DOI: 10.1103/PhysRevD.100.091501

\section{INTRODUCTION}

A deconfined system of quarks and gluons, under extreme conditions of high temperatures and high densities, can be produced and explored in experiments of ultrarelativistic heavy-ion collisions. The experimental observables like elliptic-flow measurements strongly suggest an early collective-fluid behavior of a medium close to local thermal equilibrium. However, the description of the prethermalization dynamics of the initial off-equilibrium many-body system produced in heavy-ion collisions is still an outstanding problem.

The early stage of heavy-ion collisions is well described within the color-glass-condensate (CGC) effective field theory $[1,2]$, where the heavy nuclei behave as a very dense gluon system with high energetic colored partons acting as sources of soft dynamical gluon fields. In this picture, during the collision, the hard partons traverse each other while the highly occupied soft gluon fields interact via

\footnotetext{
*lenkiewicz@th.physik.uni-frankfurt.de

thees@th.physik.uni-frankfurt.de
}

Published by the American Physical Society under the terms of the Creative Commons Attribution 4.0 International license. Further distribution of this work must maintain attribution to the author(s) and the published article's title, journal citation, and DOI. Funded by SCOAP.
non-Abelian interactions, resulting in the creation of longitudinal chromo-electric and -magnetic fields, which leads to the so-called Glasma [2-5] state of high gluon density, which runs through a very short isotropization stage [6,7]. Given the high particle density which is parametrically larger compared to the thermal-equilibrium value, the system would possess a strongly interacting nature due to coherently enhanced scattering, even though the coupling is weak. Thus, the possible formation of an off-equilibrium Bose-Einstein condensate (BEC) has drawn stronger attention in recent years $[8,9]$. Similar issues about offequilibrium BEC formations arise also in the context of early Universe reheating after inflation $[10,11]$ and in systems of cold atoms $[11,12]$.

The formation of a BEC is a fundamental consequence of quantum statistics, where above a certain critical density or below a certain critical temperature any more added bosons must occupy the ground state coherently. The condensation dynamics, especially far from equilibrium, is an interesting issue but still under debate. Many studies have been performed to understand the nonequilibrium dynamics of BECs formation within either a kinetic approach or classical field theory, if solely elastic processes are incorporated [8,13-18].

Inelastic scattering may qualitatively change the picture, allowing only for the formation of a transient BEC. In Ref. [19], it is found that inelastic collisions will speed up 
the thermalization in the infrared regime and may catalyze a faster onset of a BEC. The following study [20] suggested a complete hindrance of BEC formation for massless gluons at vanishing momentum. Within the description of a nonequilibrium massive bosonic $\mathrm{O}(N)$ theory applying the two-particle irreducible (2PI) formalism of real-time Schwinger-Keldysh quantum field theory, it has been recently shown that the formation of a BEC is potentially prevented by particle-number changing processes [21]. However, a concrete kinetic simulation for a possible transient BEC has not been included in these studies.

So far, no kinetic description has been elaborated to describe the expected transient formation and decay of a BEC, initially possible in an off-equilibrium system, including both elastic and inelastic processes. This paper addresses the dynamics of the condensation and thermalization of massive bosons. For this, a coupled set of Boltzmann kinetic equations for a transient BEC and a phase-space distribution function is formulated and includes $2 \rightarrow 2$ and particlenumber changing $2 \leftrightarrow 3$ reactions.

\section{KINETIC EQUATIONS}

In this work, we focus on an isotropic and homogeneous system. If the evolution is dominated by two- and three-body interactions, the corresponding Boltzmann equation for a phase-space distribution function $f(\vec{p})=$ $d_{g} \mathrm{~d} N /(2 \pi)^{3} \mathrm{~d}^{3} x \mathrm{~d}^{3} p$, where $d_{g}=16$ is the gluon degeneracy factor, taking two spin and eight color states into account, reads [22]

$$
\begin{aligned}
\dot{f}_{1}= & -\frac{1}{2} \frac{1}{2 E_{1}} \int \mathrm{d}^{9}(\overrightarrow{2} \overrightarrow{3} \overrightarrow{4}) \delta^{(4)}(1+2-3-4)\left\{\left|M_{12 \leftrightarrow 34}\right|^{2} f_{1} f_{2}\left(\frac{f_{3}}{d_{g}}+1\right)\left(\frac{f_{4}}{d_{g}}+1\right)-\left|M_{12 \leftrightarrow 34}\right|^{2} f_{3} f_{4}\left(\frac{f_{1}}{d_{g}}+1\right)\left(\frac{f_{2}}{d_{g}}+1\right)\right\} \\
& -\frac{1}{3 !} \frac{1}{2 E_{1}} \int \mathrm{d}^{12}(\overrightarrow{2} \overrightarrow{3} \overrightarrow{4} \overrightarrow{5}) \delta^{(4)}(1+2-3-4-5)\left\{\left|M_{12 \rightarrow 345}\right|^{2} f_{1} f_{2}\left(\frac{f_{3}}{d_{g}}+1\right)\left(\frac{f_{4}}{d_{g}}+1\right)\left(\frac{f_{5}}{d_{g}}+1\right)\right. \\
& \left.-\left|M_{345 \rightarrow 12}\right|^{2} f_{3} f_{4} f_{5}\left(\frac{f_{1}}{d_{g}}+1\right)\left(\frac{f_{2}}{d_{g}}+1\right)\right\}-\frac{1}{2 ! 2 !} \frac{1}{2 E_{1}} \int \mathrm{d}^{12}(\overrightarrow{2} \overrightarrow{3} \overrightarrow{4} \overrightarrow{5}) \delta^{(4)}(1+2+3-4-5) \\
& \times\left\{\left|M_{123 \rightarrow 45}\right|^{2} f_{1} f_{2} f_{3}\left(\frac{f_{4}}{d_{g}}+1\right)\left(\frac{f_{5}}{d_{g}}+1\right)-\left|M_{45 \rightarrow 123}\right|^{2} f_{4} f_{5}\left(\frac{f_{1}}{d_{g}}+1\right)\left(\frac{f_{2}}{d_{g}}+1\right)\left(\frac{f_{3}}{d_{g}}+1\right)\right\} .
\end{aligned}
$$

Thereby, the indices refer to the momentum phase space of the participating particles $\left(\mathrm{d} \vec{i}:=\mathrm{d}^{3} \vec{p}_{i} 2(2 \pi)^{3} E_{i}\right)$ with $E_{i}=\sqrt{p_{i}^{2}+m^{2}}$. Consequently, $f_{i}$ denotes the corresponding one-particle distribution function $f\left(t, \vec{p}_{i}\right)$. The collision integrals take into account quantum statistics via Bose enhancement factors $\left(f_{i} / d_{g}+1\right)$, leading to the correct long-time equilibrium solution for bosons. The matrix elements $|M|^{2}$ are taken as isotropic with a constant cross section, [23,24]

$$
\begin{aligned}
& \left|M_{2 \leftrightarrow 2}\right|^{2}=32 \pi s \sigma_{22}, \\
& \left|M_{2 \rightarrow 3}\right|^{2}=192 \pi^{3} \sigma_{23}, \\
& \left|M_{3 \rightarrow 2}\right|^{2}=\frac{1}{d_{g}}\left|M_{2 \rightarrow 3}\right|^{2}
\end{aligned}
$$

with $s=\left(P_{1}+P_{2}\right)^{2}$ denoting the center-momentum energy squared. Here, we point out that the interesting quantity for the simulations is the ratio of the elastic and inelastic cross sections, $\sigma_{23} / \sigma_{22}$, determining the dominating processes leading to full equilibration. Energy and particle densities are respectively given by $\epsilon_{\text {part }}(t)=\int \frac{\mathrm{d}^{3} \vec{p}}{(2 \pi)^{3}} E f(p) \quad$ and $\quad n_{\text {part }}(t)=\int \frac{\mathrm{d}^{3} \vec{p}}{(2 \pi)^{3}} f(p)$.

The general argument for the emergence of a BEC is that, if in the case of the existence of a conserved number of bosons the chemical potential converges to the mass, the distribution in the infinite-volume limit can no longer accommodate the particles in the IR regime $(p \ll m)$ although

$$
\lim _{\mu \rightarrow m} f_{\text {B.E. }}(p \ll m)=\lim _{\mu \rightarrow m} \frac{d_{g}}{\exp \left(\frac{m-\mu}{T}\right)-1}=\infty .
$$

In this case, a special treatment is necessary for the zero mode, by decomposing $f(|\vec{p}|)$ in a continuumlike part $f(|\vec{p}|>0)$ for the higher modes and a discrete part $(2 \pi)^{3} n_{\mathrm{c}}(t) \delta^{(3)}(\vec{p})$ for the zero mode [15,16,18,25].

Given any initial nonequilibrium configuration of the gluon system, one can always determine via the conservation laws if condensation has to be expected in the equilibrium limit by solving

$$
\epsilon_{\text {init }}=\epsilon_{\mathrm{eq}}(T, \mu) \quad \text { and } \quad n_{\text {init }}=n_{\mathrm{eq}}(T, \mu)
$$


and if one encounters $\mu>m$ as a solution of Eqs. (4),

$$
\begin{aligned}
\epsilon_{\text {init }} & =\epsilon_{\text {eq }}(T, \mu=m)+\epsilon_{\mathrm{c}} \text { and } \\
n_{\text {init }} & =n_{\text {eq }}(T, \mu=m)+n_{\mathrm{c}},
\end{aligned}
$$

where $\epsilon_{\mathrm{c}}$ and $n_{\mathrm{c}}$ are the energy and particle density of the condensate. Those considerations only apply for number conserving scattering processes $(2 \leftrightarrow 2)$. However, if one introduces particle-number changing $2 \leftrightarrow 3$ scattering processes, this argument breaks down for massive particles because in thermal equilibrium necessarily $\mu=0$, implying that a stable condensate cannot exist.

By inserting the ansatz $f(p)=f_{|\vec{p}|>0}+(2 \pi)^{3} n_{\mathrm{c}}(t) \delta^{(3)}(\vec{p})$ into Eq. (1), we obtain the following evolution equation for the nonzero momentum modes,

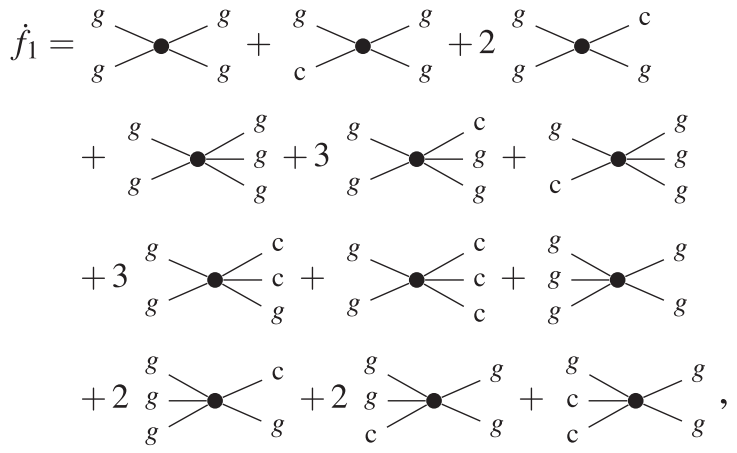

and a rate equation for the condensate density,

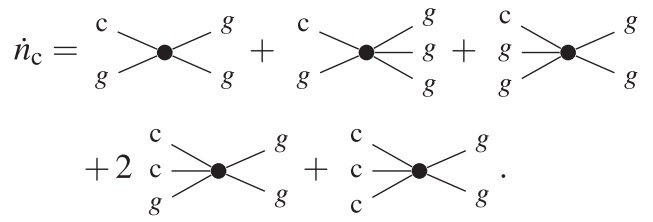

Every possible diagrammatic contribution displayed in Eqs. (6) and (7) is related to a specific collision integral, with c (condensate) and $g$ (gluon) denoting the participants of the scattering process. The numerical factors relate to the combinatorial weight of the diagrams.

For the isotropic case, the scattering angles can be integrated out analytically, leaving us with one-, two-, and three-dimensional collision integrals, which can be solved numerically. The distribution function is discretized, with the grid becoming finer in the low-momentum region. For the differential equations, we employ an efficient highorder adaptive Runge-Kutta method (Cash-Karp) [26], while the collision integrals are treated with two different integration methods. For the one- and two-dimensional integrals, we use the simple Simpson quadrature method, and for the three-dimensional integrals, we employ the VEGAS Monte Carlo integration routine from Ref. [27].

\section{INITIAL CONDITION}

In the context of the CGC framework, the two most relevant quantities are given by the saturation scale $Q_{\mathrm{s}}$ and the coupling strength $\alpha_{\mathrm{s}}$, which determines the initial population density $\propto 1 / \alpha_{\mathrm{s}}$ of the initial state. As an initial nonequilibrium isotropic profile for gluons, formed at timescales of approximately $1 / Q_{s}$, one usually considers a step function of the form $f_{\text {init }}(p)=$ $d_{g} f_{0} \Theta\left(1-p / Q_{\mathrm{s}}\right)$, whereby $f_{0} \sim 1 / \alpha_{\mathrm{s}} \quad[8,15,16,18]$. However, we use a similar function with a smooth tail around $p \approx Q_{s}$. Fixing $Q_{s}$ at $1 \mathrm{GeV}$, the only free parameter left is $f_{0}$, the step height. Various studies have shown that from this initialization two scenarios can be observed, if equilibration dynamics are dominated by binary scattering. The first is the underpopulated case $\left(f_{0}<f_{\mathrm{c}}\right)$, where the chemical potential never reaches the mass, and the second as the overpopulated case $\left(f_{0}>f_{\mathrm{c}}\right)$, where $\mu=m$ and consequently a BEC must emerge. Our investigation is focused on particles with masses $m=100(300,500) \mathrm{MeV}$ and a cross section of $\sigma_{22}=1 \mathrm{mb}$. These values are close to expected hard-thermal-loop effective pole masses of approximately $g T$ [28]. The mass acts as an effective IR regulator for the scattering or "emissions."

In nature, the initialization of the condensate is due to spontaneous fluctuations. Because we choose a deterministic approach, Eq. (7) implies $\dot{n}_{\mathrm{c}} \sim n_{\mathrm{c}}$, i.e., condensation does not occur, if $n_{\mathrm{c}}$ vanishes initially. To overcome this issue, we extract effective values for the chemical potential $\mu_{\text {eff }}$ and the temperature $T_{\text {eff }}$ by fitting the IR region $\left[f_{\mathrm{IR}}(p<m)\right]$ of the distribution function to the BoseEinstein distribution function. If now $\mu_{\text {eff }}$ approaches $m$ (let us name this point in time $t_{\text {onset }}$ ), we manually insert a finite but negligibly small seed to the zero mode $n_{\mathrm{c}}\left(t=t_{\text {onset }}\right)=10^{-6} n_{\text {init }}[15,16,18,25]$.

In the following simulations, $f_{0}=0.45(2.0)$ has been chosen such that the condensation criterion is generally fulfilled and vary in detail the ratio $\sigma_{23} / \sigma_{22}$. Our simulations start in time at $t=0$ for solving Eqs. (6) and (7).

\section{RESULTS}

In Figs. 1 and 2, the main results are depicted and compared to the known case of the evolution under solely binary scattering processes for several cross section ratios $\sigma_{23} / \sigma_{22}$. The typical overpopulated evolution for $2 \leftrightarrow 2$ interactions consists of the particle cascade toward the soft modes [Fig. 1(a)] followed by its decrease to the equilibrium distributions (e), while generating a condensate until the equilibrium is reached. The introduction of $2 \leftrightarrow 3$ kinetics will dramatically change this picture. The first observation is that the influx of particles toward the soft modes [Fig. 1(b), $\sigma_{23} / \sigma_{22}=0.0049$ ] is decelerated compared to the previous case but still sufficient to hit the onset condition somewhat later (Fig. 3), consequentially generating a condensate. But once the Bose-Einstein shape for 

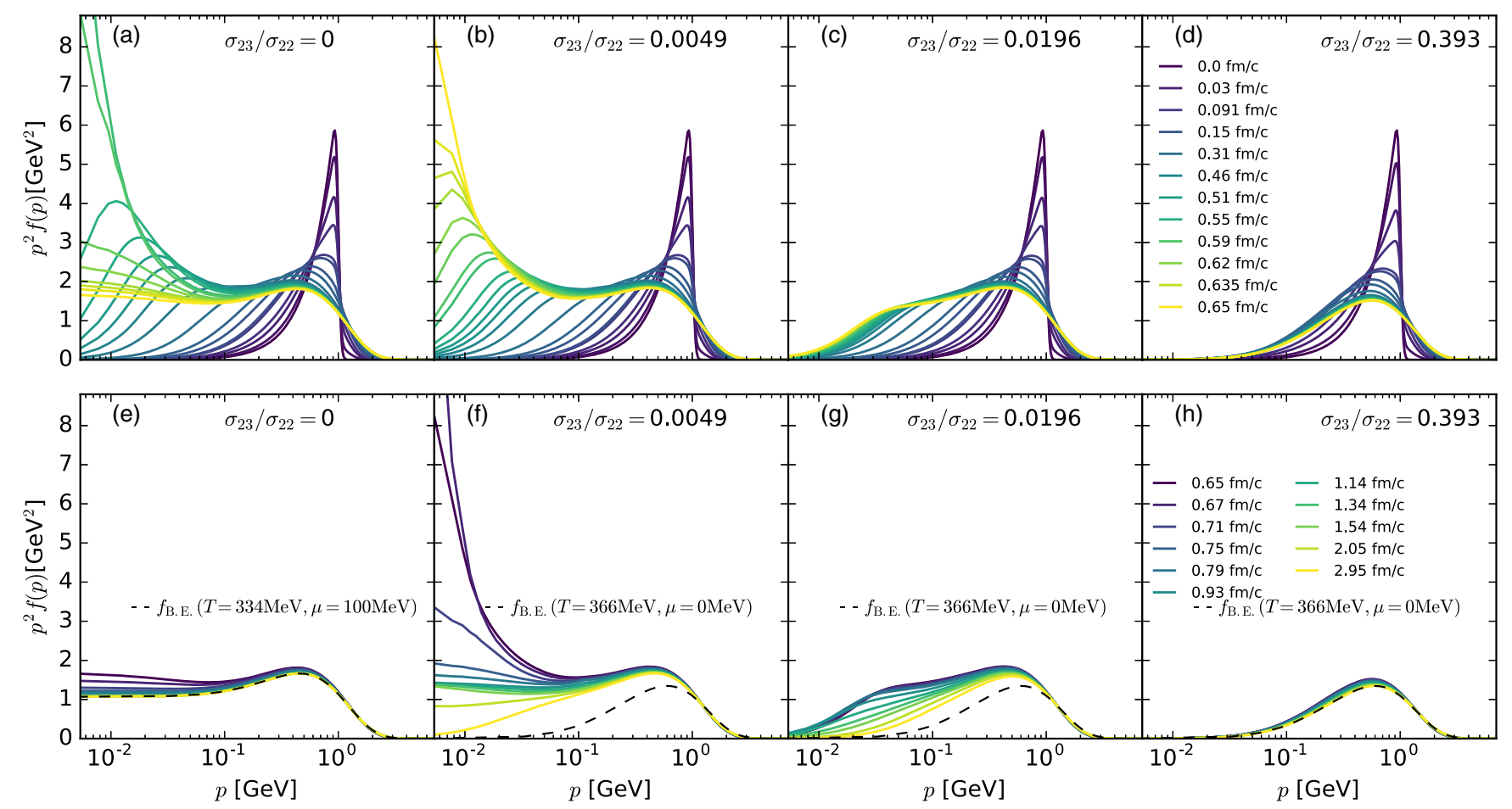

FIG. 1. Differential fraction of the isotropic particle density with respect to the momentum and for four different regimes of the cross section ratio, only binary scattering $(\mathrm{a}+\mathrm{e})$, elastically dominated scattering $(\mathrm{b}+\mathrm{f})$, balanced scattering $(\mathrm{c}+\mathrm{g})$, and inelastically dominated $(\mathrm{d}+\mathrm{h})$, with $m=100 \mathrm{MeV}$ at $f_{0}=0.45$ at various times. The rows separate two sequential time periods. In $(\mathrm{a}+\mathrm{e})$ and $(b+f)$, a condensate is present, but in (f), it decays. The black dashed lines (bottom row) depict the individual expected equilibrium states.

$\mu=m$ is recovered $[t \simeq 0.9 \mathrm{fm} / \mathrm{c}$, Figs. 1 (f) and 2], we observe that the condensate decays, contrary to the case considering only particle-number conserving $2 \leftrightarrow 2$

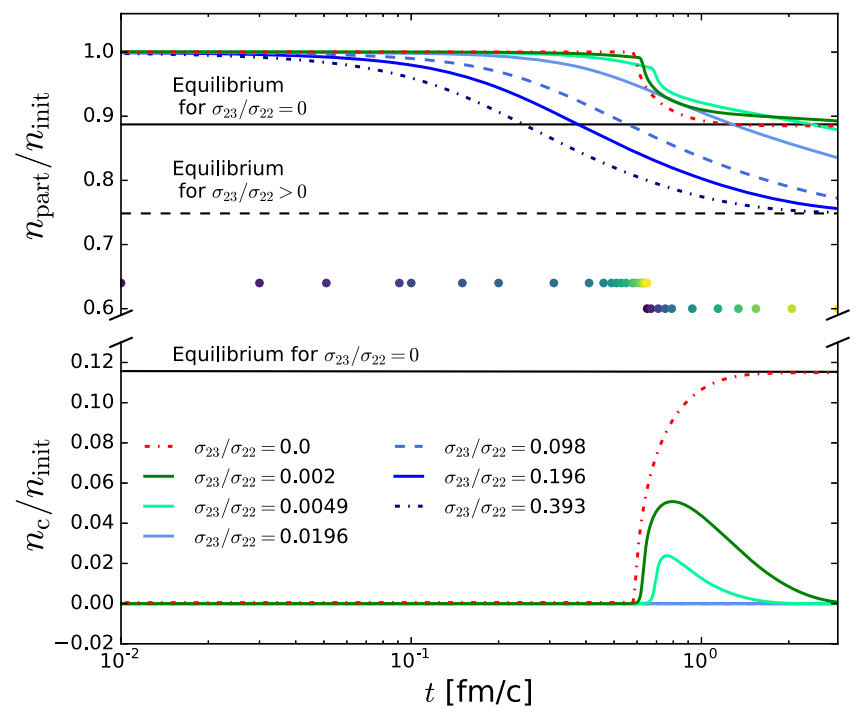

FIG. 2. Time evolution of normalized particle (upper panel) and condensate (lower panel) densities for particles with $m=$ $100 \mathrm{MeV}$ and $f_{0}=0.45$. The bluish curves show runs without the onset of condensation $\mu_{\text {eff }}<m$. The scattered dots refer to the time stamps of Fig. 1, and the black lines mark the individual expected equilibrium values. processes. For gradually larger values of $\sigma_{23} / \sigma_{22}$, the characteristic particle transport toward the soft modes is further damped, and $\mu_{\text {eff }}$ never reaches the onset of

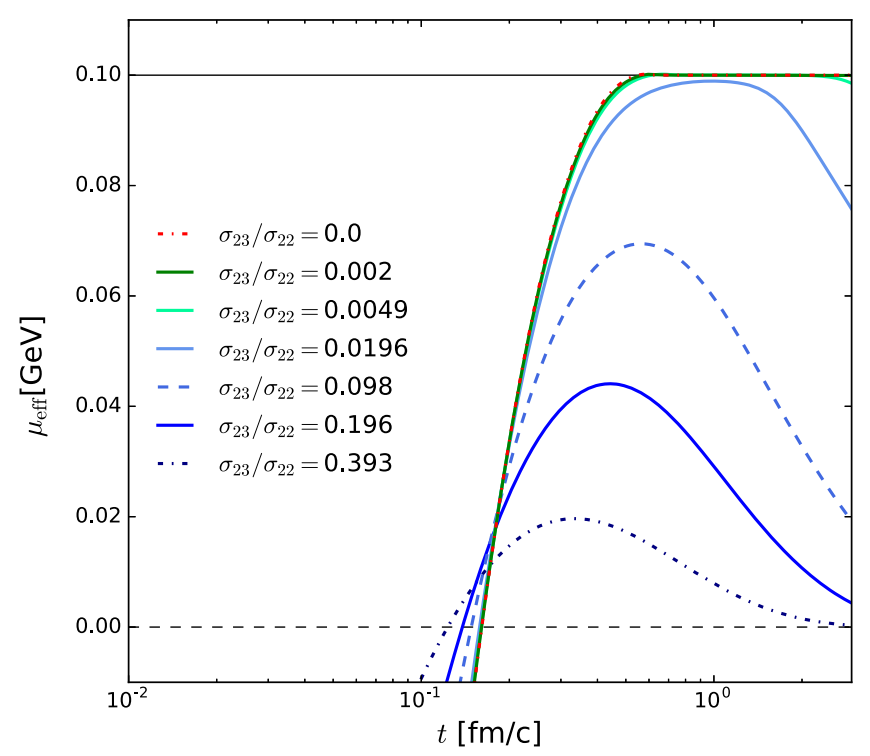

FIG. 3. Time evolution of the effective chemical potential, with $m=100 \mathrm{MeV}$ at $f_{0}=0.45$. Bluish curves (inelastic) represent runs where condensation was not feasible, contrary to the greenish (inelastic) curves and red dashed line (elastic). The black line relates to the equilibrium condition for $2 \leftrightarrow 2$ processes $(\mu=m)$, and the dashed line relates to inelastic processes $(\mu=0 \mathrm{GeV})$. 


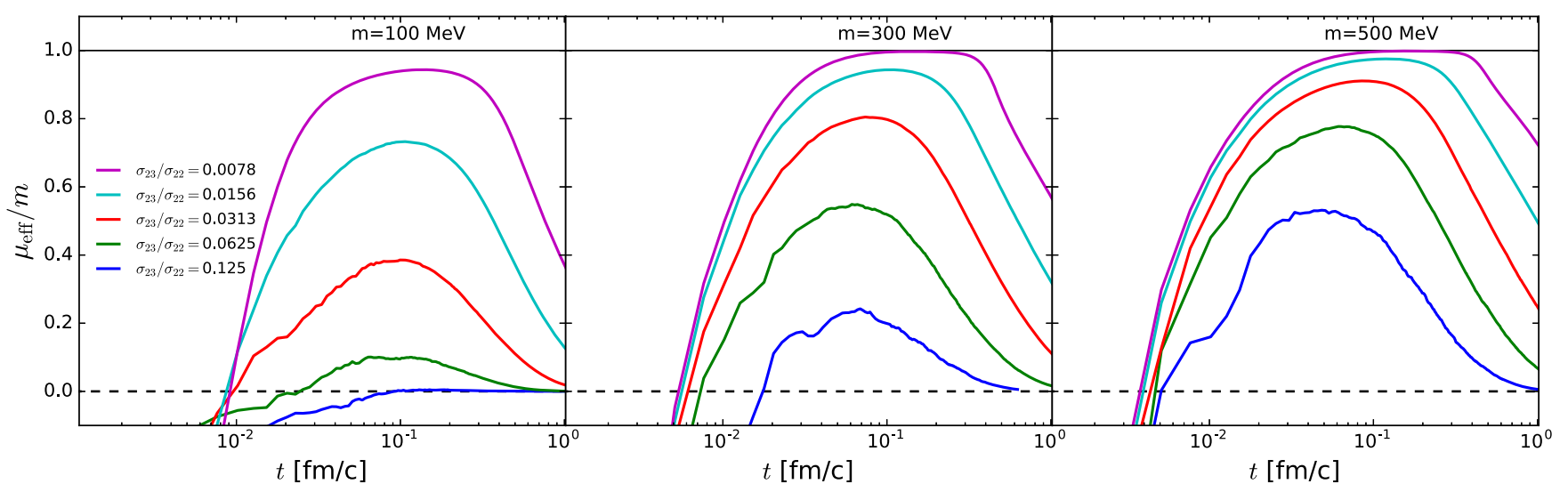

FIG. 4. Time evolution of the effective chemical potential with respect to the masses at strongly overpopulated initial condition, $f_{0}=2$, for three different masses [100 (left), 300 (middle), and $500 \mathrm{MeV}$ (right)] and for different values of $\sigma_{23} / \sigma_{22}$. Solid black lines correspond to the equilibrium condition for $2 \leftrightarrow 2$ processes, and the dashed lines correspond to the inelastic processes.

condensation. If $\sigma_{23} / \sigma_{22} \gtrsim 0.01$, then no condensation into a BEC is observed.

The situation for the chemical potential $\mu_{\text {eff }}$ can be seen in Fig. 3. While for $\sigma_{23}=0$ an equilibrium state with $\mu_{\text {eff }}=m=0.1 \mathrm{GeV}$ is reached, this is only the case for the two smallest ratios $\sigma_{23} / \sigma_{22}=0.002$ and 0.0049 , where $\mu_{\text {eff }}$ reaches $m$ but finally decreases again to reach the equilibrium state with $\mu_{\text {eff }}=0$, as is expected, if particle number is not conserved.

In Fig. 4, we show the time evolution of the effective chemical potential, $\mu_{\text {eff }}$, in dependence of various masses, $m=100,300$, and $500 \mathrm{MeV}$. Please note for these calculations we employ a strongly overpopulated initial condition with $f_{0}=2$. Inspecting the calculations, only for $\sigma_{23} / \sigma_{22}=$ 0.0078 and for masses $m=300$ and $500 \mathrm{MeV}$, the chemical potential just touches the mass limit $\mu_{\text {eff }}=m$, although no condensation will start. The effect of earlier times for the onset of BEC formation of heavier particles has also been found in a similar study with only elastic collisions [29]. Still, taking into account inelastic $2 \leftrightarrow 3$ collisions, either for smaller or larger masses, no condensation occurs for the strongly overpopulated initial condition. Only if $\sigma_{23} / \sigma_{22} \lesssim$ 0.005 , a momentary and tiny BEC can develop.

\section{CONCLUSIONS}

In this paper, we have investigated a complete BoseEinstein condensation of gluons within kinetic theory, explicitly including number changing $2 \leftrightarrow 3$ processes. An overpopulated nonequilibrium bosonic system akin to Glasma-type initial conditions has been considered. The bosons have been taken with a small but finite mass. The situation is similar to the scenario of Ref. [21]. The cross sections are not those of perturbative QCD. On the other hand, binary scatterings in thermal QCD are regulated by finite Debye-screening masses of order $O(g T)$. Radiative perturbative QCD emissions are substantial for describing the observed jet attenuation and also the significant lowering of the shear-viscosity over entropy-density ratio [30,31]. The latter fact can be effectively rephrased by significant $2 \leftrightarrow 3$ isotropic collisions [23].

Our simulations have shown that a BEC may be formed for some limited time if $\sigma_{23} / \sigma_{22} \ll 1$. For present physical parameters of the masses and overpopulation parameter, $f_{0}$, typically a BEC can only appear if $\sigma_{23}$ is less than $1 \%$ of $\sigma_{22}$. The results suggest that, as expected, particlenumber conserving and changing processes are counteracting mechanisms for the formation and destruction of a BEC. We note that the individual collision integrals scale with the occupation density of the system like approximately $f^{3}$ (elastic) and $f^{4}$ (inelastic), which resembles a sensitive scenario for possible formation but also immediate decay of a BEC.

Summarizing, our calculations show that Bose-Einstein condensates in the very early stage of heavy-ion collisions are highly unlikely if inelastic collisions are significantly participating in the dynamical gluonic evolution.

\section{ACKNOWLEDGMENTS}

We are grateful to the LOEWE Center for Scientific Computing (LOEWE-CSC) at Frankfurt for providing computing resources. We also acknowledge support by the Deutsche Forschungsgemeinschaft (DFG, German Research Foundation) through Grant No. CRC- TR 211 "Strong-interaction matter under extreme conditions," Project No. 315477589-TRR 211. Z.X.'s work was financially supported by the National Natural Science Foundation of China under Grant No. 11575092. K. Z.'s work was financially supported by the BMBF under the ErUM-Data project and the AI research grant of SAMSON AG, Frankfurt. 
[1] L. McLerran, in Proceedings of the 38th International Symposium on Multiparticle Dynamics (ISMD08), edited by J. Bartels, K. Borras, G. Gustafson, H. Jung, K. Kutak, S. Levonian, and J. Mnich (Verlag Deutsches ElektronenSynchrotron, Hamburg, 2009).

[2] F. Gelis, E. Iancu, J. Jalilian-Marian, and R. Venugopalan, Annu. Rev. Nucl. Part. Sci. 60, 463 (2010).

[3] H. Weigert, Prog. Part. Nucl. Phys. 55, 461 (2005).

[4] T. Lappi and L. McLerran, Nucl. Phys. A772, 200 (2006).

[5] D. Gelfand, A. Ipp, and D. Müller, Phys. Rev. D 94, 014020 (2016).

[6] A. Kurkela and Y. Zhu, Phys. Rev. Lett. 115, 182301 (2015).

[7] T. Epelbaum and F. Gelis, Phys. Rev. Lett. 111, 232301 (2013).

[8] J.-P. Blaizot, F. Gelis, J.-F. Liao, L. McLerran, and R. Venugopalan, Nucl. Phys. A873, 68 (2012).

[9] J.-P. Blaizot, F. Gelis, J. Liao, L. McLerran, and R. Venugopalan, Nucl. Phys. A904-A905, 829c (2013).

[10] T. Prokopec and T. G. Roos, Phys. Rev. D 55, 3768 (1997).

[11] P.-A. Pantel, D. Davesne, S. Chiacchiera, and M. Urban, Phys. Rev. A 86, 023635 (2012).

[12] R. Lacaze, P. Lalleman, Y. P. Pomeau, and S. R. Rica, Physica (Amsterdam) 152D-153D, 779 (2001).

[13] J. Berges and D. Sexty, Phys. Rev. Lett. 108, 161601 (2012).

[14] J.-P. Blaizot, J. Liao, and L. McLerran, Nucl. Phys. A920, 58 (2013).

[15] Z. Xu, K. Zhou, P. Zhuang, and C. Greiner, Phys. Rev. Lett. 114, 182301 (2015).
[16] A. Meistrenko, H. van Hees, K. Zhou, and C. Greiner, Phys. Rev. E 93, 032131 (2016).

[17] T. Epelbaum, F. Gelis, S. Jeon, G. Moore, and B. Wu, J. High Energy Phys. 09 (2015) 117.

[18] K. Zhou, Z. Xu, P. Zhuang, and C. Greiner, Phys. Rev. D 96, 014020 (2017).

[19] X.-G. Huang and J. Liao, Phys. Rev. D 91, 116012 (2015).

[20] J.-P. Blaizot, J. Liao, and Y. Mehtar-Tani, Nucl. Phys. A961, 37 (2017).

[21] S. Tsutsui, J.-P. Blaizot, and Y. Hatta, Phys. Rev. D 96, 036004 (2017).

[22] S. Weinstock, Phys. Rev. D 73, 025005 (2006).

[23] A. El, F. Lauciello, C. Wesp, Z. Xu, and C. Greiner, Nucl. Phys. A925, 150 (2014).

[24] Z. Xu and C. Greiner, Phys. Rev. C 71, 064901 (2005).

[25] D. V. Semikoz and I. I. Tkachev, Phys. Rev. Lett. 74, 3093 (1995).

[26] J. R. Cash and A. H. Karp, ACM Trans. Math. Softw. 16, 201 (1990).

[27] G. P. Lepage, J. Comput. Phys. 27, 192 (1978).

[28] A. Peshier, B. Kämpfer, O. P. Pavlenko, and G. Soff, Phys. Rev. D 54, 2399 (1996).

[29] J.-P. Blaizot, Y. Jiang, and J. Liao, Nucl. Phys. A949, 48 (2016).

[30] Z. Xu, C. Greiner, and H. Stöcker, Phys. Rev. Lett. 101, 082302 (2008).

[31] J. Uphoff, F. Senzel, O. Fochler, C. Wesp, Z. Xu, and C. Greiner, Phys. Rev. Lett. 114, 112301 (2015). 